\title{
"Let Food Be Thy Medicine": Diet and Supplements in Irritable Bowel Syndrome
}

\author{
Neha V Patel (D) \\ Digestive and Liver Diseases, Internal \\ Medicine, University of Texas \\ Southwestern Medical Center, Dallas, \\ TX, USA
}

\begin{abstract}
Overview: The purpose of this review is to introduce options for dietary therapies and supplements for the treatment of irritable bowel syndrome (IBS). IBS is a common condition with heterogeneity in pathogenesis and clinical presentation. Current treatment options are targeted at symptom relief with medications. Patients naturally pursue dietary modifications when dealing with symptoms. Dietary therapy for IBS has been poorly studied in the past; however, newer evidence suggests the use of certain diets, such as the low FODMAP (fermentable oligosaccharides, disaccharides, monosaccharides and polyols) diet, as an intervention in patients with IBS for symptom improvement. Exclusion strategies are frequently tried, such as gluten restriction or lactose avoidance, but lack quality evidence behind their use. Additionally, supplements, such as fiber, probiotics, and peppermint oil, have also been used for IBS with more recent data suggesting the use of these supplements with specific caveats.
\end{abstract}

Keywords: IBS, FODMAP, probiotics, microbiome, diarrhea, constipation, abdominal pain, bloating, gluten, sensitivity, dietary, disease, treatment

\section{Introduction}

Irritable bowel syndrome is diagnosed based on symptoms defined by the Rome IV criteria. These criteria require the presence of abdominal pain and altered bowel habits. Symptoms should be present without any significant warning signs, such as bleeding, weight loss or anemia, or nocturnal symptoms. Irritable bowel syndrome (IBS) is becoming increasingly common and costly. It is the most common condition diagnosed by gastroenterologists, affecting $11 \%$ of the world population and $12 \%$ of the US population. This condition equates to a significant financial burden in healthcare, accounting for almost 6 million prescriptions and \$20 billion in healthcare-related costs. All-cause costs have been estimated annually at $\$ 11,182$ to $\$ 13,038$ per patient. ${ }^{1}$

The pathogenesis of IBS is multifactorial and heterogeneous among patients with alterations in motility, visceral sensation, microbiome, brain-gut interaction via the central and enteric nervous systems, mucosal immune function, bile acid metabolism and intestinal permeability. More recent studies have turned attention to the interplay between the microbiome, immune function, gut sensation and motility. The microbiome of IBS patients has a different luminal bacterial composition with fewer Bifidobacteria, and Faecalibacterium prausnitzii sp and more Lactobacillaceae, Bacteroides, and Enterobacteriaceae.,3 These alterations in the microbiome cause increased visceral hypersensitivity with colonic distension in
Correspondence: Neha V Patel UT Southwestern Medical Center, 5959 Harry Hines Blvd HP5.520, Dallas, TX 75235, USA

Tel +l 214-645-6355

Email neha.patel@utsouthwestern.edu; nehavpatel@gmail.com 
animal models. IBS patients have alterations in immune activation by increase in toll-like receptor expression and production of pro-inflammatory cytokines. Degradation of tight junction proteins and increased intraepithelial permeability has been observed. This dysbiosis has effects on gut function and is an important player in the pathogenesis of IBS.

\section{Current Status of Knowledge}

Due to the heterogeneity of symptoms and multiple postulated mechanisms of disease, therapeutic options for IBS have been disappointing. Current medications for IBS target certain mechanisms such as motility, secretion or the microbiome. In randomized controlled trials (RCTs), these medications offer therapeutic gains ranging from only $8-20 \%$ over placebo, making maybe less than $1 / 2$ of patients better ${ }^{4,5}$ (Table 1).

A significant portion of patients turns to self-directed care or use alternative medicines for their symptoms. Nutritional components of food have been used medicinally for thousands of years. Hippocrates, the father of modern western medicine, was quoted: "Let food be thy medicine and medicine be thy food". Medicines derived from foods or plants have become more widely available, especially with online shopping and their presence increased in health food stores. These alternative therapies, such as probiotics and supplements, or medical foods, are not regulated by the Food and Drug Administration (FDA). They are not held to the rigorous standards in clinical trials, lending to variability among trial results. Current dietary studies have been largely of poor quality with small sample sizes, lack of blinding and randomization, and significant bias. More recently, certain diets and supplements have gained some momentum in research studies on IBS.

\section{Fiber Supplementation}

Increase in dietary fiber has been recommended for IBS by physicians for decades. Fiber has effects on colonic secretions, motility, and the microbiome. Soluble fiber mixes with water creating a gel-like matter, which is digested by bacteria and improves stool viscosity. Insoluble fiber increases stool volumes. Fiber supplementation has been adopted into practice; however, studies on fiber use had poor methodology and high risk of bias. To date, there have been 15 RCTs of fiber use in IBS in 1048 patients, which showed significant effect in global symptoms. ${ }^{6}$ There was a statistically significant benefit for the use of ispaghula or psyllium compared to placebo. Bran, which is an insoluble fiber, had no significant effect on IBS symptom relief. ${ }^{6}$ Soluble fibers, such as psyllium, wheat dextran or acacia, are reasonable low-cost intervention and recommended for patients with irritable bowel syndrome with constipation.

\section{Probiotics, Prebiotics, and Synbiotics}

Alterations in the gut microbiome contribute to the pathogenesis of IBS. This fact is supported by the development of IBS symptoms after an infection, known as postinfectious IBS. This raises the question of whether using agents to alter the microbiome improves IBS symptoms. Probiotics, prebiotics, and synbiotics have emerged as agents, which modify the microbiome for benefit to the host. Probiotics are live microorganisms, which may confer health benefits. Prebiotics are the components of food that gut microorganisms utilize to fuel the microbiome. Synbiotics are a mixture of substrates (prebiotics and live organisms) which would act synergistically to promote the growth of certain beneficial organisms. There have been

Table I Current FDA-Approved Therapies for IBS

\begin{tabular}{|c|c|c|c|c|c|}
\hline Drug Mechanism & FDA-Approved Drug & Indication & $\begin{array}{c}\text { Percentage } \\
\text { Response Drug }\end{array}$ & $\begin{array}{c}\text { Percentage } \\
\text { Response Placebo }\end{array}$ & $\begin{array}{c}\text { Number Needed to } \\
\text { Treat (NNT) }\end{array}$ \\
\hline Microbiome & Rifaximin & IBS-D & 43 & 34 & 11 \\
\hline Hypersensitivity and motility & Eluxadoline & IBS-D & 27 & 17 & 10 \\
\hline Motility & Alosetron & IBS-D & 51 & 36 & 7 \\
\hline \multirow[t]{3}{*}{ Gut Secretion } & Lubiprostone & IBS-C & 18 & 10 & 12.5 \\
\hline & Linaclotide & IBS-C & 34 & 14 & 5 \\
\hline & Plecanatide & IBS-C & 30 & 18 & 8 \\
\hline
\end{tabular}

Note: Data from these studies. ${ }^{4,5}$

Abbreviations: IBS-D, Irritable Bowel Syndrome-Diarrhea predominant; IBS-C, Irritable Bowel Syndrome- Constipation predominant. 
18 new RCTs assessing efficacy of probiotics in IBS in the past 5 years alone. ${ }^{7}$ Two RCTs on the prebiotic fructooligosaccharide versus placebo reported no significant difference in IBS symptoms with prebiotic use. One study evaluating a high-dose and low-dose prebiotic galactooligosaccharide showed significant improvement in overall symptoms and no change in abdominal pain. The two RCTs of synbiotic use in IBS were conducted in Italy and $\mathrm{S}$ Korea with both trials showing positive results without statistically significant effect in reducing symptoms. Based on the current evidence, probiotics are cautiously recommended for the improvement of symptoms, particularly bloating and flatulence, in IBS patients. There is not enough evidence to support the use of prebiotics or synbiotics. Probiotics come in many different varieties and are sold as combinations, tablet or liquids, and refrigerated forms. Combinations of probiotics, Lactobacillus sp, Bifidobacterium sp., and Streptococcus sp confer beneficial effects on IBS, yet which specific combination or species still remains unclear. ${ }^{7}$

\section{Peppermint Oil}

Peppermint oil has been used for its health benefits for many years, dating back to the 1700 s, and has been most studied in IBS. Peppermint oil has been shown to exert effects on smooth muscle calcium channels and opioid receptors, leading to muscle relaxation, changes in contractions or motility, and alteration of pain sensitivity. A systematic review and meta-analysis of five randomized controlled trials of peppermint oil (197 patients on peppermint oil and 195 on placebo) favored its use over placebo (RR $2.23(95 \%$ CI 1.78 to 2.81$)) .{ }^{8}$ Global improvement in IBS symptoms and abdominal pain was seen in patients receiving peppermint oil compared to placebo. These effects on pain improvement are quickacting, modest, and typically short-lived. Peppermint oil is an alternative modality used in IBS patients for pain control, with the caveat that the data is based on few small studies with specific formulations of the supplement.

\section{Dietary Therapy for IBS}

The vast majority of IBS patients perceive their symptoms as related to either specific foods or meal intake in general. ${ }^{9}$ While many patients jump to the conclusion that this implies a food allergy, there are many potential explanations for such postprandial exacerbations of symptoms, most of which represent activation of physiologic rather than allergic responses. Foods with a high content of fat and spice are thought to give rise to GI symptoms. When surveying 197 Swedish patients with IBS about foods as triggers for their symptoms, the culprit foods were those containing carbohydrates and histamine. Contrary to popular belief, fatty foods were less commonly identified as triggers. Histamine and other biogenic amines occur in fish, fermented foods, vegetables, wine and beer. High levels of histamines can provoke symptoms such as nausea, abdominal pain and diarrhea. Alternatively, foods may not contain a significant amount of histamine but may trigger an endogenous histamine release. Still, patients cannot help relating their symptoms to food intake and continue to search for triggers. Patients try different strategies to manage their symptoms, and they often do it without any medical guidance. A survey of 1500 Gastroenterologists across the US found that $50 \%$ of patients try a form of exclusion diet based on food triggers, and a $1 / 4$ of patients have tried a gluten-free diet (GFD). ${ }^{10}$ Dietary modification is ubiquitous and commonplace in clinical practice.

\section{Exclusion Diets Based on Testing}

Many patients keep food diaries or try exclusion diets based on triggers. When rechallenged with the foods they perceive as triggers, the majority of patients do not report exacerbation of symptoms. Certain commercially available tests are based on gut immune activation of specific foods and claim to identify food intolerances; however, these tests are not validated. The tests can be quite costly (up to $\$ 1000$ per panel) with poor coverage by commercial providers. IgG-based food allergy panel testing is frequently requested by patients and performed with insufficient data to support this practice. Foodrelated IgG4 testing is also available, but only indicates repeated exposure to foods, and does not indicate allergy, intolerance, nor hypersensitivity. IgG or IgG4 Food-allergy testing is not recommended by the GI and Allergy societies. Exclusion diet based on leukocyte antibody testing (LAT) of peripheral blood or the ALCAT test was studied in one trial in IBS patients. Fifty-eight patients randomized to an exclusion diet based on LAT or sham diet reported improvement in symptoms; however, no significant difference was seen in overall quality of life and adequate relief of symptoms. ${ }^{11}$ These results spark interest in immune activation in IBS and larger studies are needed. 


\section{Lactose Restriction}

Many IBS patients attribute their symptoms to milk or dairy intolerance. This self-reported intolerance does not correlate with objective evidence of malabsorptions, such as hydrogen breath testing. Older poor-quality trials reported robust symptom response to lactose restriction. This response may be a consequence of lactase deficiency, yet a study providing lactase supplementation to IBS patients did not show improvement in their symptoms. ${ }^{12}$ There are no blinded or controlled trials, which show the benefit of lactose restriction. Hence, there is no highquality evidence to support the use of lactose restriction in IBS patients.

\section{Gluten Restriction}

Some patients attribute their IBS symptoms to gluten or wheat ingestion. Attention to gluten-related conditions, including Celiac Disease and gluten intolerance or allergy, has increased dramatically, reflecting the marked growth of the gluten-free food industry. This has extended to the population of healthy individuals who believe that adhering to a GFD may have benefits on overall health regardless of GI symptoms. The prevalence of people who avoid gluten has more than tripled from 2009 to 2014, with nearly 3 million people following a GFD. ${ }^{13}$ There are two well-recognized clinical entities where GI symptoms are triggered by gluten, which may present with symptoms similar to IBS. Celiac Disease (CD) is an enteropathy where gluten exposure to genetically predisposed individuals with HLA DQ2 or DQ8 genotype results in inflammation characterized by infiltration of the lamina propria and epithelium with lymphocytes and villous atrophy. Patients have elevated serum biomarkers and may have extra-intestinal manifestations, such as a rash called dermatitis herpetiformis or vitamin deficiencies or osteoporosis. Non-celiac gluten sensitivity (NCGS) is an entity where patients have symptoms related to gluten intake and improve on a gluten-free diet without any genetic predisposition or serum biomarker elevation or enteritis. The pathogenesis of NCGS has some hallmarks of innate immune system activation; however, this has not been clearly defined. Since patients with Celiac Disease and NCGS present with symptoms paralleling IBS, it is of utmost importance to exclude CD due to long-term implications, such as malignancy, infertility, and osteoporosis. What is alarming, though, is that $62 \%$ of adults who report an intolerance to gluten do not have appropriate biomarkers and testing for $\mathrm{CD}$ before initiating a glutenfree diet. ${ }^{14}$

Gluten-free diets may help certain IBS patients. A double-blinded RCT exposed patients without Celiac Disease who previously responded to gluten-free diets to either gluten or placebo diets. Of the 19 patients in the gluten arm, $68 \%$ of patients reported uncontrolled symptoms compared to $40 \%$ of patients in the placebo arm. Patients exposed to gluten complained of worsening pain, bloating, stool consistency, and fatigue. ${ }^{15}$ While other trials suggest improved outcomes for patients on gluten-free diets, there are only two small randomized controlled trials assessing gluten-free diets in IBS involving 111 participants. Both trials reported statistically significant results; however, in a pooled analysis, the results were not statistically significant. ${ }^{16}$ Perhaps, a more personalized approach may increase the efficacy of gluten restriction in IBS patients. One study explored the effects of gluten-free or glutencontaining diets on patients with diarrhea-predominant IBS. A gluten-containing diet was associated with increased bowel movements and small bowel permeability, especially in those positive for HLA DQ8 or DQ2. ${ }^{17}$ Patients with diarrhea-predominant symptoms may have a better response. Another explanation for the unconvincing evidence of a gluten-free diet is that perhaps the issue is not gluten alone but other components of wheat products causing symptoms. Fructans are a polymer of fructose molecules that are present in many foods and grains including wheat, barley, rye, onions, and garlic. Wheat also contains other proteins called amylase trypsin inhibitors, which can exacerbate GI symptoms. To test this hypothesis, a study from Norway took 59 patients with self-reported gluten sensitivity and randomized them to fructan diet, gluten diet, or placebo for one week. ${ }^{12}$ Results demonstrated significantly higher symptom scores after fructan intake than gluten intake or placebo. The problem may not be gluten specific, but rather the fructans in wheat, which lead to symptoms.

\section{Low FODMAP Diet}

A group in Australia developed a diet that focused on lowering the amount of poorly absorbed, fermentable carbohydrates collectively, called the low FODMAP diet.

FODMAPs are fermentable oligo-, di-, and monosaccharides and polyols. Oligosaccharide-containing foods are onions, shallots, garlic, barley, cabbage, artichoke, asparagus, beans and chickpeas. Foods containing monosaccharides like fructose include apples, pears, watermelon, and mango. Examples of polyols are sorbitol, mannitol, and 
other artificial sweeteners. FODMAPs are characterized by limited small intestinal absorption and are broken down by bacteria into short-chain fatty acids (SCFAs). SCFAs create high osmotic activity, which increases intestinal secretions and water content and lowers luminal $\mathrm{pH}$. In healthy individuals, functional MRI testing after ingesting fructose showed increased small bowel water content. ${ }^{18}$ Ingestion of fructose and inulin, which is a fructan, also led to increased hydrogen breath production and increased colonic luminal distension. These effects rarely cause symptoms in healthy subjects. When coupled with visceral hypersensitivity in IBS patients, these effects have the capability of inducing significant GI symptoms. High FODMAP foods exert effects on the colon through bacterial fermentation, which produces higher levels of hydrogen and methane gas. IBS patients on high FODMAP diets have been shown to produce higher levels of breath hydrogen than healthy volunteers and higher breath hydrogen levels than IBS patients on a low FODMAP diet. ${ }^{19}$ FODMAPs induce effects on the small intestine and colon, which lead to pain, gas/bloating, and altered bowel movements in IBS patients (Figure 1).

There are growing data, which supports the use of the low FODMAP diet (LFD) in IBS. Seven randomized controlled trials evaluated 397 participants on a low FODMAP diet compared to either an alternate sham or placebo diet, an IBS diet based on NICE guidelines, or high FODMAP diet. The LFD was effective in improving IBS symptoms with an RR of continued symptoms on a low FODMAP diet of 0.69 (95\% CI 0.54 to 0.88 ). The NNT for the FODMAP diet was 5 (95\% CI 3 to 11$).{ }^{20}$ More recently, a multi-center questionnaire study revealed that $76 \%$ of patients will continue the personalized LFD even long term (mean follow-up $>3.5$ years). ${ }^{21}$ Another study compared the effects of a low
FODMAP diet to an IBS diet based on NICE (National Institute for Health and Care) guidelines in patients with diarrhea-predominant symptoms. There was a higher number of abdominal pain responders in the LFD group compared to the NICE diet ( $51 \%$ vs $23 \%, P=0.008) .{ }^{22}$ However, there was no significant difference in adequate relief of symptoms or the composite end point between the groups. Compared with baseline scores, the low FODMAP diet led to greater reductions in average daily scores of abdominal pain, bloating, consistency, frequency, and urgency. More studies report IBS symptom improvement on the low FODMAP diet. ${ }^{23-25}$

High FODMAP food elimination is only a first step towards treatment. The elimination period lasts for 4-6 weeks, which is viewed as a diagnostic test to identify patients sensitive to FODMAPs. Those who fail to improve do not continue the diet. Those with response should be instructed on reintroducing foods containing FODMAPs gradually to identify their sensitivities. This information should then be used to liberalize and personalize their diet to minimize symptoms. The reintroduction phase is a crucial step in the process and should be done under the guidance of a dietician for long-term success. ${ }^{21,24}$ The FODMAP diet can limit calcium intake significantly to less than the daily recommended intake $(<800 \mathrm{mg} / \mathrm{d})$ during the elimination phase. ${ }^{26}$ It also limits fiber intake. While there may be slight differences in micronutrient intake, such as thiamine and riboflavin, during the diet, these are still within the normal range and not significant clinically.

The FODMAP diet also has the ability to alter immune regulation in the gut. Hustoft and colleagues reported improved IBS symptoms and decreased pro-inflammatory cytokines IL-6 and IL-8 in their double-blinded RCT of LFD. $^{27}$ In another study, urinary histamine, a marker of

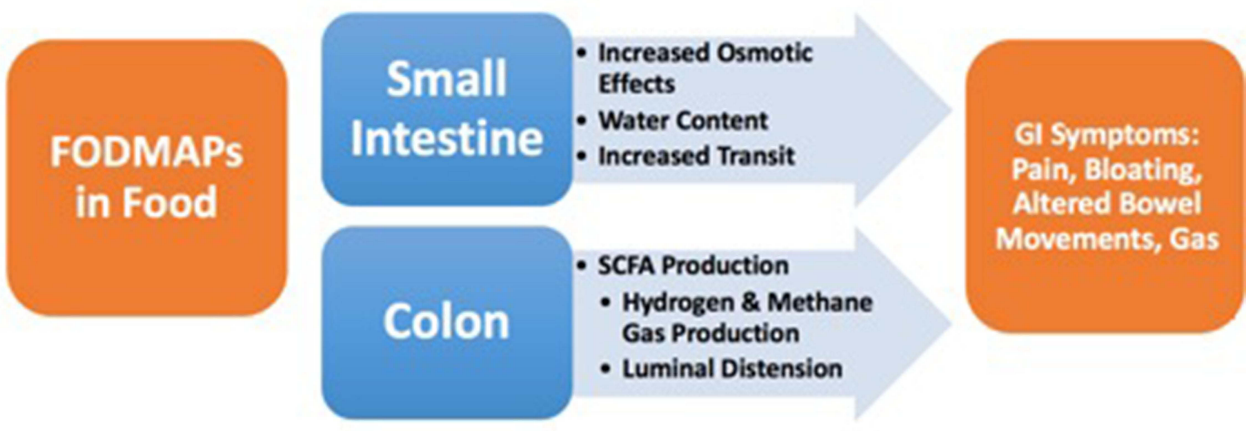

Figure I FODMAPs in foods have effects on the small intestine and colon which lead to symptoms in Irritable Bowel Syndrome patients. Abbreviations: FODMAP, fermentable oligosaccharides, disaccharides, monosaccharides and polyols; SCFA, short-chain fatty acids; GI, gastrointestinal. 
immune activation, decreased 8 -fold in those on the low FODMAP diet. ${ }^{28}$ The diet alters the microbiome with a reduction in concentration of species and a decrease in total bacteria. Certain beneficial species, Bifidobacteria, Actinobacteria and Faecalibacterium prausnitzii, were less abundant in IBS patients on the low FODMAP diet, which some speculate to be a result of reducing dietary prebiotic components. These effects are short-lived as several of these bacteria rebounded after 10 days of highfructooligosaccharide (high FODMAP) supplementation. ${ }^{27}$

Currently, there are no identifiable characteristics that predict response to the diet. A Norwegian study analyzed gut microbial composition as a marker for the response to FODMAP diet by assessing 54 fecal bacterial markers with 16s rRNA sequencing. Responders had higher level of markers for Bacteroides fragilis, Acinetobacter, Ruminiclostridium, Streptococcus and Eubacterium and lower levels of markers for Clostridia, Actinomycetes, and Shigella/Escherichia. ${ }^{29}$ Another study measured volatile organic compounds in gases emanated from stool in patients on a low FODMAP diet. Results showed that certain patterns of volatile organic compounds could identify patients who were responders to the LFD or a probiotic supplement with a mean accuracy of $97 \%$ and $89 \%$, respectively. ${ }^{30}$ Perhaps, gut microbiota composition or VOC (volatile organic compound) profiling may be developed as tools to identify responses to dietary FODMAP restriction; however, further studies are necessary.

Implementation and adherence to the diet can be an arduous task for many patients. Removal of dairy products, wheat products, certain fruits and vegetables and sweeteners can make food options quite limited. Physicians lack the time and sometimes the expertise in counseling patients on diet modifications. ${ }^{10}$ Referral to a dietician is highly recommended when feasible. If the dietician visit is not covered by insurance, patients can use online support via websites, such as myginutrition.com and even apps, such as the Monash FODMAP app to identify foods. ${ }^{31}$ Many low FODMAP recipe books and blogs by registered dieticians are available online, which can guide patients on meal preparation.

\section{Conclusion}

Fiber supplementation, dietary changes, use of peppermint oil and probiotics are all strategies in the toolbox for treatment of IBS patients. The low FODMAP diet has been viewed by gastroenterologists as a primary strategy for therapy in IBS. Many GIs consider the diet as effective, if not better, than current medical treatments. ${ }^{10}$ The American College of Gastroenterology has recommended a LFD for a limited time in the guidelines for management of IBS for overall symptom improvement based on the currently available data. ${ }^{4}$ They recommended against the use of gluten-free diets or exclusion diets based on antibody or leukocyte activation testing. ${ }^{20}$ Personalized dietary modification, ie gluten or wheat avoidance or diet based on testing, still needs further study. Identifying patients with a higher likelihood of response to dietary or supplement therapy is also an area of growing interest. Dietary strategies in IBS are becoming more evidencebased than in earlier practice and are now a primary strategy for therapy in IBS, similar to other chronic disease conditions, such as diabetes or cardiovascular disease.

\section{Statement of Significance}

Dietary therapies and supplements have a role in the management of patients with Irritable Bowel Syndrome. The current review of the evidence supports the use of the LFD (Low-FODMAP diet) and soluble fiber supplementation, and cautious use of probiotics in certain patients.

\section{Acknowledgments}

A preliminary version of this paper was presented at the University of Texas Southwestern Internal Medicine Grand Rounds conference with interim findings. The poster's abstract was published in Internal Medicine Grand Rounds Protocols on the UT Southwestern Grand Rounds website: [https:// utswmed-ir.tdl.org/bitstream/handle/2152.5/6882/123 071219_Protocol_PatelN.pdf?sequence=1\&isAllowed=y]”.

\section{Funding}

There is no funding to report.

\section{Disclosure}

Neha V Patel, M.D. does not have any financial interests or other relationships with commercial concerns related directly or indirectly to this journal.

\section{References}

1. Buono JL, Mathur K, Averitt AJ, Andrae DA. Economic Burden of Irritable Bowel Syndrome with Diarrhea: retrospective Analysis of a U.S. Commercially Insured Population. J Manag Care Spec Pharm. 2017;23(4):453-460.

2. Liu $\mathrm{HN}, \mathrm{Wu} \mathrm{H}$, Chen $\mathrm{Y}-\mathrm{Z}$, et al. Altered molecular signature of intestinal microbiota in irritable bowel syndrome patients compared with healthy controls: a systematic review and meta-analysis. Dig Liver Dis. 2017;49(4):331-337. doi:10.1016/j.dld.2017.01.142 
3. Pittayanon R, Lau JT, Yuan Y, et al. Gut Microbiota in Patients With Irritable Bowel Syndrome-A Systematic Review. Gastroenterology. 2019;157(1):97-108. doi:10.1053/j.gastro.2019.03.049

4. Lacy BE, Pimentel M, Brenner DM, et al. ACG Clinical Guideline: management of Irritable Bowel Syndrome. Am J Gastroenterol. 2021;116(1):17-44. doi:10.14309/ajg.0000000000001036

5. Chey WD. Food: the Main Course to Wellness and Illness in Patients With Irritable Bowel Syndrome. Am J Gastroenterol. 2016;111 (3):366-371. doi:10.1038/ajg.2016.12

6. Nagarajan N, Morden A, Bischof D, et al. The role of fiber supplementation in the treatment of irritable bowel syndrome: a systematic review and meta-analysis. Eur J Gastroenterol Hepatol. 2015;27 (9):1002-1010. doi:10.1097/MEG.0000000000000425

7. Ford AC, Harris LA, Lacy BE, et al. Systematic review with meta-analysis: the efficacy of prebiotics, probiotics, synbiotics and antibiotics in irritable bowel syndrome. Aliment Pharmacol Ther. 2018;48(10):1044-1060. doi:10.1111/apt.15001

8. Khanna R, MacDonald JK, Levesque BG. Peppermint oil for the treatment of irritable bowel syndrome: a systematic review and meta-analysis. J Clin Gastroenterol. 2014;48(6):505-512. doi:10.10 97/MCG.0b013e3182a88357

9. Bohn L, Störsrud S, Liljebo T, et al. Diet low in FODMAPs reduces symptoms of irritable bowel syndrome as well as traditional dietary advice: a randomized controlled trial. Gastroenterology. 2015;149 (6):1399-1407 e2. doi:10.1053/j.gastro.2015.07.054

10. Lenhart A, Ferch C, Shaw M, et al. Use of Dietary Management in Irritable Bowel Syndrome: results of a Survey of Over 1500 United States Gastroenterologists. J Neurogastroenterol Motil. 2018;24 (3):437-451. doi:10.5056/jnm17116

11. Ali A, Weiss TR, McKee D, et al. Efficacy of individualised diets in patients with irritable bowel syndrome: a randomised controlled trial. BMJ Open Gastroenterol. 2017;4(1):e000164. doi:10.1136/bmjgast2017-000164

12. Lisker R, Solomons NW, Briceño RP, Mata MR. Lactase and placebo in the management of the irritable bowel syndrome: a double-blind, cross-over study. Am J Gastroenterol. 1989;84(7):756-762.

13. Choung RS, Unalp-Arida A, Ruhl CE, et al. Less Hidden Celiac Disease But Increased Gluten Avoidance Without a Diagnosis in the United States: findings From the National Health and Nutrition Examination Surveys From 2009 to 2014. Mayo Clin Proc. 2016;92:30-38.

14. Biesiekierski JR, Newnham ED, Shepherd SJ, et al. Characterization of Adults With a Self-Diagnosis of Nonceliac Gluten Sensitivity. Nutr Clin Pract. 2014;29(4):504-509. doi:10.1177/088453361452 9163

15. Biesiekierski JR, Newnham ED, Irving PM, et al. Gluten causes gastrointestinal symptoms in subjects without celiac disease: a double-blind randomized placebo-controlled trial. Am J Gastroenterol. 2011;106 (3):508-14; quiz 515. doi:10.1038/ajg.2010.487

16. Dionne J, Ford AC, Yuan Y, et al. A Systematic Review and Meta-Analysis Evaluating the Efficacy of a Gluten-Free Diet and a Low FODMAPs Diet in Treating Symptoms of Irritable Bowel Syndrome. Am J Gastroenterol. 2018;113(9):1290-1300. doi:10.10 38/s41395-018-0195-4

17. Vazquez-Roque MI, Camilleri M, Smyrk T, et al. A controlled trial of gluten-free diet in patients with irritable bowel syndrome-diarrhea: effects on bowel frequency and intestinal function. Gastroenterology. 2013;144(5):903-911 e3. doi:10.1053/ j.gastro.2013.01.049
18. Murray K, Wilkinson-Smith V, Hoad C, et al. Differential effects of FODMAPs (fermentable oligo-, di-, mono-saccharides and polyols) on small and large intestinal contents in healthy subjects shown by MRI. Am J Gastroenterol. 2014;109(1):110-119. doi:10.1038/ajg.2013.386

19. Ong DK, Mitchell SB, Barrett JS, et al. Manipulation of dietary short chain carbohydrates alters the pattern of gas production and genesis of symptoms in irritable bowel syndrome. J Gastroenterol Hepatol. 2010;25(8):1366-1373. doi:10.1111/j.1440-1746.2010.06370.x

20. Ford AC, Moayyedi P, Chey WD, et al. American College of Gastroenterology Monograph on Management of Irritable Bowel Syndrome. Am J Gastroenterol. 2018;113(Suppl 2):1-18. doi:10.1038/s41395-018-0084-x

21. Rej A, Shaw CC, Buckle RL, et al. The low FODMAP diet for IBS; A multicentre UK study assessing long term follow up. Dig Liver Dis. 2021. doi:10.1016/j.dld.2021.05.004

22. Eswaran SL, Chey WD, Han-Markey T, et al. A Randomized Controlled Trial Comparing the Low FODMAP Diet vs. Modified NICE Guidelines in US Adults with IBS-D. Am J Gastroenterol. 2016;111(12):1824-1832. doi:10.1038/ajg.2016.434

23. Zhang Y, Feng L, Wang X, et al. Low fermentable oligosaccharides, disaccharides, monosaccharides, and polyols diet compared with traditional dietary advice for diarrhea-predominant irritable bowel syndrome: a parallel-group, randomized controlled trial with analysis of clinical and microbiological factors associated with patient outcomes. Am J Clin Nutr. 2021;113(6):1531-1545.

24. Goyal O, Batta S, Nohria S, et al. Low fermentable oligosaccharide, disaccharide, monosaccharide, and polyol diet in patients with diarrhea-predominant irritable bowel syndrome: a prospective, randomized trial. J Gastroenterol Hepatol. 2021;36(8):2107-2115. doi:10.1111/jgh. 15410

25. Zahedi MJ, Behrouz V, Azimi M. Low fermentable oligo-di-monosaccharides and polyols diet versus general dietary advice in patients with diarrhea-predominant irritable bowel syndrome: a randomized controlled trial. J Gastroenterol Hepatol. 2018;33(6):1192-1199. doi:10.1111/jgh.14051

26. Eswaran S, Dolan RD, Ball SC, Jackson K, Chey W. The Impact of a 4-Week Low-FODMAP and mNICE Diet on Nutrient Intake in a Sample of US Adults with Irritable Bowel Syndrome with Diarrhea. J Acad Nutr Diet. 2019.

27. Hustoft TN, Hausken T, Ystad SO, et al. Effects of varying dietary content of fermentable short-chain carbohydrates on symptoms, fecal microenvironment, and cytokine profiles in patients with irritable bowel syndrome. Neurogastroenterol Motil. 2017;29(4):e12969. doi:10.1111/nmo.12969

28. McIntosh K, Reed DE, Schneider T, et al. FODMAPs alter symptoms and the metabolome of patients with IBS: a randomised controlled trial. Gut. 2017;66(7):1241-1251. doi:10.1136/gutjnl-2015-311339

29. Valeur J, Småstuen MC, Knudsen T, et al. Exploring Gut Microbiota Composition as an Indicator of Clinical Response to Dietary FODMAP Restriction in Patients with Irritable Bowel Syndrome. Dig Dis Sci. 2018;63(2):429-436. doi:10.1007/s10620-017-4893-3

30. Rossi M, Aggio R, Staudacher HM, et al. Volatile Organic Compounds in Feces Associate With Response to Dietary Intervention in Patients With Irritable Bowel Syndrome. Clin Gastroenterol Hepatol. 2018;16 (3):385-391 e1. doi:10.1016/j.cgh.2017.09.055

31. Eswaran S, Farida JP, Green J, et al. Nutrition in the management of gastrointestinal diseases and disorders: the evidence for the low FODMAP diet. Curr Opin Pharmacol. 2017;37:151-157. doi:10.1016/j.coph.2017.10.008 


\section{Publish your work in this journal}

Clinical and Experimental Gastroenterology is an international, peerreviewed, open access, online journal publishing original research, reports, editorials, reviews and commentaries on all aspects of gastroenterology in the clinic and laboratory. This journal is indexed on American Chemical Society's Chemical Abstracts Service (CAS).
The manuscript management system is completely online and includes a very quick and fair peer-review system, which is all easy to use. Visit http://www.dovepress.com/testimonials.php to read real quotes from published authors.

Submit your manuscript here: https://www.dovepress.com/clinical-and-experimental-gastroenterology-journal 